\begin{tabular}{|c|c|c|}
\hline \multirow{2}{*}{$\begin{array}{r}\text { Case Reports in } \\
\text { Gastroenterology }\end{array}$} & \multicolumn{2}{|c|}{ Case Rep Gastroenterol 2013;7:63-68 } \\
\hline & $\begin{array}{l}\text { DOI: } 10.1159 / 000348758 \\
\text { Published online: March 1, } 2013\end{array}$ & $\begin{array}{l}\text { (c) } 2013 \text { S. Karger AG, Basel } \\
1662-0631 / 13 / 0071-0063 \$ 38.00 / 0 \\
\text { www.karger.com/crg }\end{array}$ \\
\hline
\end{tabular}

\title{
Pediatric Gastric Volvulus: Diagnostic and Clinical Approach
}

\author{
Federica Porcaro ${ }^{a} \quad$ Girolamo Mattioli $^{\mathrm{b}}$ Claudio Romano ${ }^{\mathrm{a}}$ \\ ${ }^{a}$ Pediatric Department, University of Messina, Messina, and ${ }^{b}$ Department of Pediatric \\ Surgery, Giannina Gaslini Institute, Genova, Italy
}

\section{Key Words}

Postprandial vomiting · Chronic gastric volvulus · Anterior and fundal gastropexy

\begin{abstract}
Gastric volvulus is a significant, rare cause of non-bilious vomiting and consists of a pathological rotation of the stomach of more than $180^{\circ}$ around the axis without obstruction of the gastrointestinal tract. A definitive diagnosis is made with upper radiological gastrointestinal studies. Treatment may be conservative or surgical with anterior and fundal gastropexy in patients with ingravescent symptoms. We describe the case of a 16-month-old female admitted to our hospital for recurrent and postprandial vomiting episodes which had started at 11 months of age. A history of gastroesophageal reflux was present until 1 year of age, in association with recurrent respiratory infections. The basic metabolic panel was normal. Barium study showed stomach rotation along a horizontal plane stomach. Esophagogastroduodenoscopy showed no mucosal alterations. The diagnosis was chronic organoaxial gastric volvulus. In our patient, the surgical procedure of gastropexy, both anterior and fundal, without fundoplication was performed. She showed good improvement after surgery, with resolution of symptoms and weight gain.
\end{abstract}

\section{Introduction}

Gastric volvulus (GV) is a significant, rare cause of non-bilious vomiting and consists of a pathological rotation of the stomach of more than $180^{\circ}$ around the axis, which causes obstruction of the gastrointestinal tract. A rotation of the stomach of $<180^{\circ}$ that leads to only partial foregut obstruction is defined as 'gastric torsion'. It is usually described with a peak incidence during the fifth decade of life [1] and is generally considered rare in the pediatric population. According to anatomic localization, GV may be classified into intraabdominal and intrathoracic. The last one is infrequent in the pediatric age group and is usually related with

Claudio Romano

Pediatric Department, University of Messina

Via Osservatorio 1, IT-98122 Messina (Italy)

E-Mail romanoc@unime.it 
diaphragmatic hernia with subsequent dislocation of the stomach in the thorax. Gastric rotation can occur on its longitudinal (organoaxial) or transverse (mesenteroaxial) axis. Stomach rotation about both its longitudinal or transverse axes is defined as 'combined volvulus' [2]. Organoaxial volvulus is the most common variant in the pediatric age group. GV may be associated with anatomic defects of gastric ligaments (idiopathic or primary GV), with abnormalities of gastric anatomy and function, or with abnormalities of adjacent organs (secondary GV) (table 1) [3]. GV is a clinical condition that may present in either acute or chronic type. Anatomic defects are usually associated with acute GV; otherwise, absence or laxity of the anchoring ligaments of the stomach represent predisposing factors to chronic GV.

The diagnosis of chronic GV is often delayed, and for this reasons it should be suspected in children with a history of chronic vomiting, abdominal distension, failure to thrive and recurrent chest infection. A definitive diagnosis is made with upper gastrointestinal studies, particularly using barium study that reveals gastric rotation and estimates the amount of obstruction. The treatment varies depending on the type of GV. Acute GV often requires immediate surgical treatment; otherwise, chronic GV should be treated conservatively (prone position with head slightly up, prokinetics and antisecretory drugs). Nevertheless, surgical treatment of chronic GV (both anterior and fundal gastropexy) remains controversial and limited to patients with persistent or severe symptoms. The present case underlines the importance of clinical and diagnostic management of children with chronic vomiting: the diagnostic and clinical approach should be personalized based on the content and the features of vomiting, as well as the persistence of alarm signals such us failure to thrive.

\section{Case Report}

A 16-month-old female was admitted to our hospital for recurrent and postprandial vomiting episodes which had started at 11 months of age, occurring, on average, 6 times per day. Feeding problems resulting from recurrent vomiting were associated with failure to thrive. A history of gastroesophageal reflux unresponsive to drug therapy was present until 1 year of age, in association with recurrent respiratory infections. On physical exam, weight was $9.97 \mathrm{~kg}\left(<50^{\circ} \mathrm{P}\right)$ and height $82.5 \mathrm{~cm}\left(<95^{\circ} \mathrm{P}\right)$. Her general condition at admission was good, with abdominal bloating in the absence of organomegaly. We performed routine basic metabolic panel with: glycemia $(84 \mathrm{mg} / \mathrm{dl}$ ), liver (serum glutamic oxaloacetic transaminase $12 \mathrm{IU} / \mathrm{dl}$, serum glutamic pyruvate transaminase $18 \mathrm{IU} / \mathrm{dl}$ ) and renal (azotemia $32 \mathrm{mg} / \mathrm{dl}$, creatinine $0.3 \mathrm{mg} / \mathrm{dl}$ ) function tests, complete blood count (red blood count $3.9 \times 10^{6} / \mathrm{mm}^{3}$, hemoglobin $10.8 \mathrm{~g} / \mathrm{dl}$, platelets $\left.237 \times 10^{3} / \mathrm{mm}^{3}\right)$ and white blood cell count $\left(7 \times 10^{3} / \mathrm{mm}^{3}\right.$, neutrophils $54 \%$, eosinophils $2 \%$, basophils $0 \%$, lymphocytes $38 \%$, monocytes $6 \%$ ), C-reactive protein $(0.4 \mathrm{mg} / \mathrm{dl})$, amylase $(41 \mathrm{U} / \mathrm{l})$ and lipase $(15 \mathrm{U} / \mathrm{l})$, serum ammonia (52 $\mu \mathrm{mol} / \mathrm{l})$, lactate $(13 \mathrm{mg} / \mathrm{dl})$, muscle enzymes (creatine phosphate kinase $47 \mathrm{IU} / \mathrm{ml}$, lactate dehydrogenase $250 \mathrm{U} / \mathrm{l}$ ), basal cortisol $(122 \mathrm{ng} / \mathrm{ml}$ ), and thyroid hormones (free thyroxine $1.7 \mathrm{ng} / \mathrm{dl}$, thyroid-stimulating hormone $1.2 \mathrm{mU} / \mathrm{ml}$ ). These results can be considered normal for the patient's age. Also, plasma amino acids, celiac serology, urinalysis, cytomegalovirus serology, coproculture and parasitological examination of stools were negative. Neurological examination with electroencephalogram and fundus oculi were normal. Barium study showed stomach rotation along a horizontal plane stomach with partial rotation of the greater curvature to the diaphragmatic side; the stomach was markedly dilated with delayed gastric emptying (fig. 1, fig. 2). Esophagogastroduodenoscopy showed 
Porcaro et al.: Pediatric Gastric Volvulus: Diagnostic and Clinical Approach

no mucosal alterations. The diagnosis was chronic organoaxial GV with a difficult decision between a conservative approach and a possible surgical solution.

\section{Discussion}

GV is a rare condition that is defined as a pathological rotation of the stomach. It was first described by Berti in 1866. While acute GV is very rare, chronic GV is being diagnosed with increasing frequency. Both acute and chronic forms have distinctive patterns of presentation. Clinical presentation includes symptoms such as chest infection, failure to thrive and gastroesophageal reflux (table 2). Clinical cases with mild or moderate symptoms should be treated conservatively, while those with persistent and severe symptoms should undergo anterior and fundal gastropexy without fundoplication. The natural history of chronic GV is poorly understood. It can transform into acute volvulus with specific symptoms including severe epigastric pain and vomiting. Overall, $40 \%$ of infants with chronic GV are treated successfully with conservative management and subsequent spontaneous improvement of symptoms with growth and development. In children, acute GV is frequently associated with other anatomic defects, such as diaphragmatic hernia, eventration of the diaphragm, paraesophageal hernia and congenital asplenia [4]. In contrast, chronic GV is predominantly idiopathic and only in a minority of cases is it associated with anatomic or functional anomalies of the stomach or adjacent organs.

The diagnosis of chronic GV is difficult; it requires a high index of suspicion and a confirmatory upper gastrointestinal barium study showing the stomach lying horizontal and upside down. Plain radiography might be diagnostic in a few cases, but generally fails to reveal GV below the diaphragm [5]. Abdominal sonography is not helpful, as upper gastrointestinal endoscopy cannot be regarded as a diagnostic tool. The treatment may be medical or surgical depending on the underlying etiology of the volvulus. Otherwise, management of each chronic GV child should be carefully individualized. It is important for clinicians to take note of the patient's age, comorbidity, physical performance and presence of failure to thrive [6]. Conservative or non-operative management is associated with therapeutic agent use such as prokinetics, anti-secretory therapy and diet modifications (thickening of meals). It also includes position of the infant on his/her right side or in the prone position after feeding [6].

Al-Salem [7] reported successful conservative treatment in 11 pediatric patients with chronic GV, and concluded that those with mild to moderate symptoms should be treated conservatively. Acute GV can develop into chronic GV and lead to strangulation, necrosis and perforation of the stomach requiring life-saving resuscitation [8]. The mortality rate for acute GV is more than twice that of chronic GV and can occur as an abdominal emergency requiring immediate surgical treatment [4]. Laparoscopic surgery can be regarded as the treatment of choice, but conservative treatment appears to be a safe alternative. Randomized trials might be necessary to elucidate the most appropriate management in chronic GV with definition of different surgical risks of the patients.

In our case, non-bilious vomiting and growth failure were the most important symptoms suggestive of chronic or intermittent type GV. This symptomatology was ingravescent over the years, for which reason the surgical procedure of gastropexy, both anterior (to the abdominal wall) and fundal (to the diaphragm), without fundoplication was performed. The patient showed good improvement after surgery with resolution of symptoms and weight gain. 
Porcaro et al.: Pediatric Gastric Volvulus: Diagnostic and Clinical Approach

In conclusion, chronic GV is a condition documentable with barium studies in children with a history of vomiting. The approach is always conservative in the absence of alarm signals. Only in selected cases can the worsening of symptoms and the association with failure to thrive be an indication for a definitive surgical option.

\section{Disclosure Statement}

The authors declare that they have no competing interests.

\section{Authors' Contributions}

Federica Porcaro and Claudio Romano managed the patient and prepared the case report. Girolamo Mattioli is the surgeon who shared the surgical choice.

\section{References}

1 Gourgiotis S, Vougas V, Germanos S, Baratsis S: Acute gastric volvulus: diagnosis and management over 10 years. Dig Surg 2006;23:169-172.

2 Eek S, Hagelsteen H: Torsion of the stomach as a cause of vomiting in infancy. Lancet 1958;1(7010):26-28.

-3 Wasselle JA, Norman J: Acute gastric volvulus: pathogenesis, diagnosis, and treatment. Am J Gastroenterol 1993;88:1780-1784.

4 Cribbs RK, Gow KW, Wulkan ML: Gastric volvulus in infant and children. Pediatrics 2008;122:e752-e762.

5 Menuck L: Plain film findings of gastric volvulus herniating into the chest. AJR Am J Roentgenol 1976;126: 1169-1174.

-6 Hsu YC, Perng CL, Chen CK, Tsai JJ, Lin HJ: Conservative management of chronic gastric volvulus: 44 cases over 5 years. World J Gastroenterol 2010;16:4200-4205.

7 Al-Salem AH: Acute and chronic gastric volvulus in infants and children: who should be treated surgically? Pediatr Surg Int 2007;23:1095-1099.

8 Carter R, Brewer LA 3rd, Hinshaw DB: Acute gastric volvulus. A study of 25 cases. Am J Surg 1980;140:99106. 
Porcaro et al.: Pediatric Gastric Volvulus: Diagnostic and Clinical Approach

Table 1. Etiological classification of GV [5]

Idiopathic or primary GV

Anatomic defects of gastric ligaments

Gastrocolic ligament

Gastrohepatic ligament

Gastrophrenic ligament

Gastrosplenic ligament

Secondary GV

Anatomic defects or function abnormalities of the stomach

Gastric distension (prevented gastric emptying, hypomotility, aerophagia)

Hourglass stomach (congenital or secondary to neoplasm or peptic ulcer disease)

Gastric ptosis

Anatomic defects or function abnormalities of adjacent organs

Diaphragm (discontinuous surface, palsy)

Spleen (numerical or size abnormalities, wandering spleen)

Liver (dislocation or hypoplasia of left lobe)

Intestinal malrotation

Transverse colon (rotation, intrathoracic dislocation)

Table 2. Patterns of presentation of acute and chronic GV [5]

Acute volvulus

Non-bilious emesis

Abdominal distension

Abdominal pain

Acute respiratory distress

Cyanosis

Hematemesis

Apnea

Failure to thrive

Dyspnea
Chronic volvulus

Non-bilious emesis

Abdominal distension

Failure to thrive

Abdominal pain

Gastroesophageal reflux

Colic

Hematemesis

Apnea

Dyspnea

Cyanosis 


\section{Case Reports in \\ Gastroenterology}

\begin{tabular}{l|l}
\hline Case Rep Gastroenterol 2013;7:63-68 \\
\hline DOI: $\underline{10.1159 / 000348758}$ & $\begin{array}{l}@ 2013 \text { S. Karger AG, Basel } \\
\text { www.karger.com/crg }\end{array}$ \\
\hline
\end{tabular}

Porcaro et al.: Pediatric Gastric Volvulus: Diagnostic and Clinical Approach

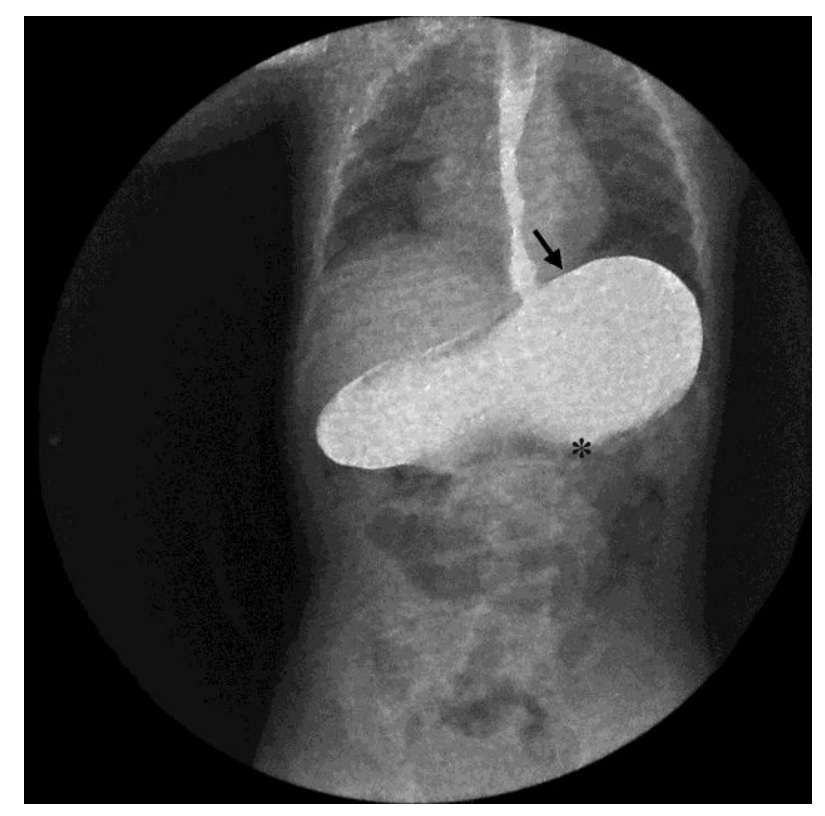

Fig. 1. Chronic organoaxial volvulus. Barium study shows inversion of the greater (arrow) and lesser curvatures (asterisk) of the stomach and gastric rotation along a horizontal plane.

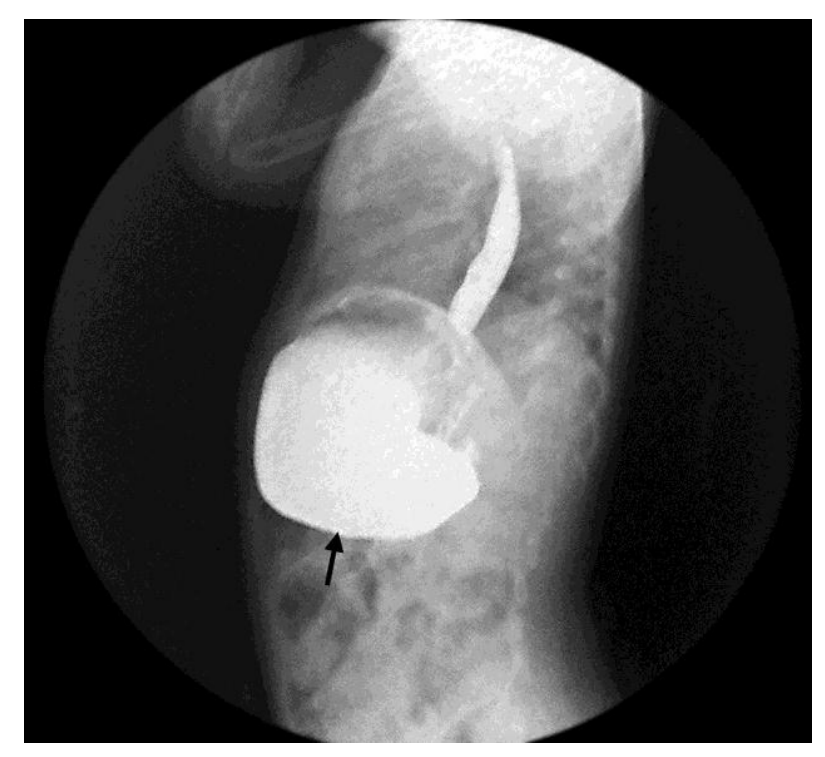

Fig. 2. Chronic organoaxial volvulus. Barium study shows marked distension of the stomach in the lateral view (arrow). 